\title{
Existence and Stability for Stochastic Partial Differential Equations with Infinite Delay
}

\author{
Jing Cui, ${ }^{1}$ Litan Yan, ${ }^{2}$ and Xichao Sun ${ }^{3}$ \\ ${ }^{1}$ Department of Mathematics, Anhui Normal University, 1 East Beijing Road, Wuhu 241000, China \\ ${ }^{2}$ Department of Mathematics, Donghua University, 2999 North Renmin Road, Songjiang, Shanghai 201620, China \\ ${ }^{3}$ Department of Mathematics and Physics, Bengbu College, Bengbu 233030, China
}

Correspondence should be addressed to Jing Cui; jcui123@126.com

Received 9 November 2013; Accepted 17 December 2013; Published 8 January 2014

Academic Editor: Weilin Xiao

Copyright (c) 2014 Jing Cui et al. This is an open access article distributed under the Creative Commons Attribution License, which permits unrestricted use, distribution, and reproduction in any medium, provided the original work is properly cited.

We consider a class of neutral stochastic partial differential equations with infinite delay in real separable Hilbert spaces. We derive the existence and uniqueness of mild solutions under some local Carathéodory-type conditions and also exponential stability in mean square of mild solutions as well as its sample paths. Some known results are generalized and improved.

\section{Introduction}

The theory of stochastic partial differential equations (SPDEs) has recently become an important area of investigation stimulated by its numerous applications to problems arising in natural and social sciences. There is much current interest in studying qualitative properties for SPDEs (see, e.g., Caraballo et al. [1], Liu [2], Luo and Liu [3], Da Prato and Zabczyk [4], Peszat and Zabczyk [5], Wang and Zhang [6], and references therein). We would like to mention that the stochastic partial functional differential equations (SPFDEs) have been considered intensively. For example, under the global Lipschitz and linear growth conditions, Govindan [7] showed by the stochastic convolution the existence, uniqueness, and almost sure exponential stability of neutral SPDEs with finite delays; Taniguchi et al. [8] considered the existence and uniqueness of mild solutions to SPDEs with finite delays by Banach fixed point theorem; while by imposing a so-called Carathéodory condition on the nonlinearities, Jiang and Shen [9] studied the existence and uniqueness of mild solutions for neutral SPFDEs by successive approximation; Samoilenko et al. [10] investigated the existence, uniqueness, and controllability results for neutral SPFDEs.

On the other hand, it is well known that infinite delay (stochastic) equations have wide application in many areas
$[11,12]$. However, as for neutral SPDEs with infinite delay, as far as we know, there exist only a few results about the existence and asymptotic behavior of mild solutions. We mention here the recent papers by Ren and Sun [13] and Li and Liu [14] considering the existence of solutions of secondorder stochastic evolution equations and neutral stochastic differential inclusions with infinite delay, respectively; Cui and Yan [15] investigated the existence and longtime behavior of mild solutions for a class of neutral stochastic partial differential equations with infinite delay in distribution, while Taniguchi [16] concerned the existence and asymptotic behavior for stochastic evolution equations with infinite delay.

In this paper, inspired by the aforementioned papers $[13,15]$, we consider a class of neutral stochastic partial differential equations (NSPDEs) with infinite delay. The space $\mathscr{B}((-\infty, 0], \llbracket)$ (see Section 2$)$ with some axioms proposed by Hale and Kato [17] is employed as our phase space. We study the existence and uniqueness of mild solutions to SPDEs with infinite delay under some local Carathéodory conditions with the non-Lipschitz conditions in Bao and Hou [18] and Jiang and Shen [9] being regarded as special cases and investigate the longtime behavior of mild solutions as well.

The structure of this paper is as follows. In the next section, we introduce some necessary notations and preliminaries. The existence and uniqueness of mild solutions are 
discussed in Section 3. The exponential stability in mean square of mild solutions as well as its sample paths are presented in Section 4.

\section{Preliminaries}

For more details on this section, we refer to Da Prato and Zabczyk [4] and Pazy [19]. Let $\left(\mathbb{H},|\cdot|_{\mathbb{\square}},\langle\cdot, \cdot\rangle_{\mathbb{\square}}\right)$ and $\left(\mathbb{K},|\cdot|_{\mathbb{K}},\langle\cdot, \cdot\rangle_{\mathbb{K}}\right)$ be two separable Hilbert spaces. $\mathscr{L}(\mathbb{K}, \mathbb{M})$ stands for the set of all linear bounded operators from $\mathbb{K}$ into $\mathbb{M}$, equipped with the usual operator norm $\|\cdot\|$. In this paper, we use the symbol $\|\cdot\|$ to denote norms of operators regardless of the spaces involved when no confusion possibly arises.

Let $\left(\Omega, \mathscr{F},\left\{\mathscr{F}_{t}\right\}_{t \geq 0}, P\right)$ be a filtered complete probability space satisfying the usual condition, which means that the filtration is a right continuous increasing family and $\mathscr{F}_{0}$ contains all $P$-null sets. Let $W=\left(W_{t}\right)_{t \geq 0}$ be a $\mathbb{K}$-valued Wiener process defined on $\left(\Omega, \mathscr{F},\left\{\mathscr{F}_{t}\right\}_{t \geq 0}, P\right)$ with covariance operator Q; that is,

$$
E\langle w(t), x\rangle_{\mathbb{K}}\langle w(s), y\rangle_{\mathbb{K}}=(t \wedge s)\langle Q x, y\rangle_{\mathbb{K}}, \quad x, y \in \mathbb{K},
$$

where $Q$ is a positive, self-adjoint, trace class operator on $\mathbb{K}$. Denote by $\mathscr{L}_{2}^{0}(\mathbb{K}, \mathbb{H})$ the space of all Q-Hilbert-Schmidt operators from $\mathbb{K}$ to $\mathbb{W}$ with the norm

$$
|\xi|_{\mathscr{L}_{2}^{0}}^{2}:=\operatorname{tr}\left(\xi Q \xi^{*}\right)<\infty, \quad \xi \in \mathscr{L}(\mathbb{K}, \mathbb{W}) .
$$

Let $A$ be the infinitesimal generator of an analytic semigroup $S(t)$ in $\mathbb{H}$. Then $(A-\alpha I)$ is invertible and generates a bounded analytic semigroup for $\alpha>0$ large enough. Suppose that $0 \in \rho(A)$, where $\rho(A)$ denotes the resolvent set of $A$. Then, for $\alpha \in(0,1]$, it is possible to define the fractional power operator $(-A)^{\alpha}$ as a closed linear invertible operator on its domain $\mathscr{D}\left((-A)^{\alpha}\right)$. Furthermore, the subspace $\mathscr{D}\left((-A)^{\alpha}\right)$ is dense in $\mathbb{H}$ and the expression

$$
\|x\|_{\alpha}=\left\|(-A)^{\alpha} x\right\|, \quad x \in \mathscr{D}\left((-A)^{\alpha}\right),
$$

defines a norm on $\mathbb{H}_{\alpha}:=\mathscr{D}\left((-A)^{\alpha}\right)$.

Throughout this paper, we will employ an axiomatic definition of the phase space $\mathscr{B}$ introduced by Hale and Kato [17].

Definition 1. The axioms of the phase space $\mathscr{B}((-\infty, 0], \mathbb{H})$ (denoted by $\mathscr{B}$ simply) are established for $\mathscr{F}_{0}$-measurable, continuous functions mapping $(-\infty, 0]$ into $\mathbb{H}$ endowed with a norm $\|\cdot\|_{\mathscr{B}}$, and $\mathscr{B}$ satisfies the following axioms:

$\left(H_{1}\right)$ If $x:(-\infty, T] \rightarrow \mathbb{H}, T>0$, is continuous on $[0, T]$ and $x_{0} \in \mathscr{B}$, then, for every $t \in[0, T]$, the following properties hold:

(1) $x_{t}:=x(t+\cdot) \in \mathscr{B}$;

(2) $|x(t)| \leq H\left\|x_{t}\right\|_{\mathscr{B}}$;

(3) $\left\|x_{t}\right\|_{\mathscr{B}} \leq M(t) \sup _{0 \leq s \leq t}|x(s)|+N(t)\left\|x_{0}\right\|_{\mathscr{B}}$, where $H>0$ is a constant, $M, N:[0,+\infty) \rightarrow$ $[1,+\infty)$ are independent of $x(\cdot)$, and $M$ is continuous and $N$ is locally bounded.

$\left(H_{2}\right)$ The space $\mathscr{B}$ is complete.
Remark 2. For convenience, we replace condition (3) in $\left(H_{1}\right)$ by

$$
\begin{aligned}
& \left(3^{\prime}\right)\left\|x_{t}\right\|_{\mathscr{B}} \leq \sup _{0 \leq s \leq t}|x(s)|+N\left\|x_{0}\right\|_{\mathscr{B}} \text {, where } N= \\
& \sup _{0 \leq s \leq T} N(s) .
\end{aligned}
$$

Example 3. Let $\left(\mathbb{H},|\cdot|_{\mathbb{Q}}\right)$ be a Banach space and $\varphi \in$ $C((-\infty, 0], \mathbb{H})$. Assume that $h:(-\infty, 0] \rightarrow(0,+\infty)$ is a continuous function with $l=\int_{-\infty}^{0} h(t) d t<+\infty$. Define

$$
\begin{gathered}
|\varphi|_{\mathbb{Q}}=\sup _{\theta \leq 0}\left(E|\varphi(\theta)|^{2}\right)^{1 / 2}<\infty, \\
\mathscr{C}_{h}=\{\varphi:(-\infty, 0] \longrightarrow \mathbb{H}, \\
\left.\int_{-\infty}^{0} h(s) \sup _{s \leq \theta \leq 0}\left(E|\varphi(\theta)|^{2}\right)^{1 / 2} d s<+\infty\right\} .
\end{gathered}
$$

If $\mathscr{C}_{h}$ is endowed with the norm

$$
\|\varphi\|_{\mathscr{C}_{h}}=\int_{-\infty}^{0} h(s) \sup _{s \leq \theta \leq 0}\left(E|\varphi(\theta)|^{2}\right)^{1 / 2} d s, \quad \varphi \in \mathscr{C}_{h}
$$

then $\left(\mathscr{C}_{h},\|\cdot\|_{\mathscr{C}_{h}}\right)$ is a Banach space [14] and satisfies the axioms in Definition 1 with $M=l, N=1$.

Consider the following NSPDEs with infinite delay in the form:

$$
\begin{aligned}
d\left[x(t)+G\left(t, x_{t}\right)\right]= & {\left[A x(t)+b\left(t, x_{t}\right)\right] d t } \\
& +\sigma\left(t, x_{t}\right) d W(t), \quad t \geq 0, \\
x(t)= & \phi(t) \in \mathscr{B}, \quad t \leq 0,
\end{aligned}
$$

where $x_{t}=\{x(t+\theta):-\infty<\theta \leq 0\}$ can be regarded as a $\mathscr{B}$-valued stochastic process. Assume that

$$
G, f: \mathbb{R}^{+} \times \mathscr{B} \longrightarrow \mathbb{H}, \quad \sigma: \mathbb{R}^{+} \times \mathscr{B} \longrightarrow \mathscr{L}(\mathbb{K}, \mathbb{H})
$$

are appropriate mappings specified later. The initial value $\phi=$ $\{\phi(\theta):-\infty<\theta \leq 0\}$ is an $\mathscr{F}_{0}$-measurable $\mathscr{B}$-valued random variable independent of $W$ with finite second moment.

Now we present the definition of the mild solution for (6).

Definition 4. An $\mathscr{F}_{t}$-adapted $\mathbb{U}$-valued stochastic process $x(t)$ defined on $-\infty<t \leq T, 0 \leq T<\infty$ is called the mild solution for (6) if

(a) $x(t)$ is continuous and $\left\{x_{t}: 0 \leq t \leq T\right\}$ is a $\mathscr{B}$-valued stochastic process;

(b) $\int_{0}^{T}|x(u)|_{\mathbb{\sharp}}^{2} d u<\infty$ almost surely; 
(c) for arbitrary $t \in[0, T], x(t)$ satisfies the following integral equation:

$$
\begin{aligned}
x(t)= & S(t)(\phi(0)+G(0, \phi))-G\left(t, x_{t}\right) \\
& -\int_{0}^{t} A S(t-s) G\left(s, x_{s}\right) d s+\int_{0}^{t} S(t-s) b\left(s, x_{s}\right) d s \\
& +\int_{0}^{t} S(t-s) \sigma\left(s, x_{s}\right) d W(s),
\end{aligned}
$$$$
x_{0}=\phi \in \mathscr{B} \text {. }
$$

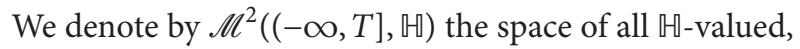
continuous, and $\mathscr{F}_{t}$-adapted processes $x=\{x(t),-\infty<t \leq$ $T\}$ such that

(1) $x_{0}=\phi \in \mathscr{B}$ and $x(t)$ is continuous on $[0, T]$;

(2) for all $x \in \mathscr{M}^{2}((-\infty, T], \mathbb{H})$,

$$
\|x\|_{\mathscr{M}^{2}}^{2}:=E\|\phi\|_{\mathscr{B}}^{2}+E \int_{0}^{T}|x(t)|_{\mathbb{\llbracket}}^{2} d t<\infty .
$$

It is obvious that the space $\mathscr{M}^{2}((-\infty, T], \mathbb{U})$ is a Banach space with the norm defined by (9).

\section{The Existence and Uniqueness Theorem}

In this section, we present our main results on the existence and uniqueness of the mild solution of (6). We first introduce the following assumptions.

$\left(A_{1}\right)$ Assume that $A$ is the infinitesimal generator of an analytic semigroup of bounded linear operators $\{S(t), t \geq$ $0\}$ in $\mathbb{U}$, satisfying

$$
\|S(t)\| \leq e^{-\gamma t}, \quad t \geq 0
$$

for some $\gamma>0$.

$\left(A_{2}\right)$ There exist some constants $\alpha \in(1 / 2,1]$ and $M_{G}>$ 0 such that, for any $\xi, \phi \in \mathscr{B}, t \geq 0$, we have $G(t, \xi), G(t, \phi) \in \mathscr{D}\left((-A)^{\alpha}\right)$, and

$$
\left|(-A)^{\alpha} G(t, \xi)-(-A)^{\alpha} G(t, \phi)\right|_{\mathbb{\square}} \leq M_{G}\|\xi-\phi\|_{\mathscr{B}},
$$

we further assume that $G(t, 0) \equiv 0$, for all $t \geq 0$.

$\left(A_{3}\right) \quad$ (a) There exists a function $F: \mathbb{R}^{+} \times \mathbb{R}^{+} \rightarrow \mathbb{R}^{+}$such that $F(t, u)$ is locally integrable in $t$ for any fixed $u \geq 0$ and is continuous, nondecreasing, and concave in $u$ for each fixed $t \in[0, T]$. Moreover, for any $t \in[0, T], x \in \mathscr{B}$, the following inequality holds:

$$
|b(t, x)|_{\square}^{2}+|\sigma(t, x)|_{\mathscr{L}_{2}^{0}}^{2} \leq F\left(t,\|x\|_{\mathscr{B}}^{2}\right) .
$$

(b) For any $C>0$, the differential equation

$$
\frac{d u}{d t}=C F(t, u)
$$

has a global solution for any initial value $u_{0}$.

\section{$\left(A_{4}\right)$ (global conditions)}

(a) There exists a function $Z: \mathbb{R}^{+} \times \mathbb{R}^{+} \rightarrow \mathbb{R}^{+}$such that $Z(t, u)$ is locally integrable in $t$ for any fixed $u \geq 0$ and is continuous nondecreasing and concave in $u$ for each fixed $t \in[0, T], Z(t, 0)=0$ for any $t \in[0, T]$. Moreover, for any $t \in[0, T]$, $x, y \in \mathscr{B}$, the following inequality holds:

$$
\begin{aligned}
& |b(t, x)-b(t, y)|_{\mathbb{Q}}^{2}+|\sigma(t, x)-\sigma(t, y)|_{\mathscr{L}_{2}^{0}}^{2} \\
& \quad \leq Z\left(t,\|x-y\|_{\mathscr{B}}^{2}\right) .
\end{aligned}
$$

(b) For any constant $D>0$, if a nonnegative function $u(t)$ satisfies that

$$
\begin{gathered}
u(t) \leq D \int_{0}^{t} Z(s, u(s)) d s, \quad t \in[0, T], \\
\text { then } u(t) \equiv 0 \text { for any } t \in[0, T] .
\end{gathered}
$$

$\left(A_{4}^{\prime}\right)$ (local conditions)

(a) For any integer $N>0$, there exists a function $Z_{N}: \mathbb{R}^{+} \times \mathbb{R}^{+} \rightarrow \mathbb{R}^{+}$such that $Z_{N}(t, u)$ is locally integrable in $t$ for any fixed $u \geq 0$ and is continuous nondecreasing and concave in $u$ for each fixed $t \in[0, T], Z_{N}(t, 0)=0$ for any $t \in[0, T]$. Moreover, for any $t \in[0, T], x, y \in \mathscr{B}$ with $\|x\|_{\mathscr{B}} \leq N,\|y\|_{\mathscr{B}} \leq N$, the following inequality holds:

$$
\begin{aligned}
& |b(t, x)-b(t, y)|_{\mathbb{\boxplus}}^{2}+|\sigma(t, x)-\sigma(t, y)|_{\mathscr{L}_{2}^{0}}^{2} \\
& \quad \leq Z_{N}\left(t,\|x-y\|_{\mathscr{B}}^{2}\right) .
\end{aligned}
$$

(b) For any constant $D>0$, if a nonnegative function $u(t)$ satisfies that

$$
\begin{gathered}
u(t) \leq D \int_{0}^{t} Z_{N}(s, u(s)) d s, \quad t \in[0, T], \\
\text { then } u(t) \equiv 0 \text { for any } t \in[0, T] .
\end{gathered}
$$

The following lemma that appeared in [19] is useful.

Lemma 5. Under the assumption of $\left(A_{1}\right)$, for any $0<\beta \leq 1$, the following equality holds:

$$
S(t)(-A)^{\beta} x=(-A)^{\beta} S(t) x, \quad x \in \mathscr{D}\left((-A)^{\beta}\right),
$$

and there exists a positive constant $M_{\beta}$ such that, for any $t>0$,

$$
\left\|(-A)^{\beta} S(t)\right\| \leq M_{\beta} t^{-\beta} e^{-\gamma t} .
$$

Lemma 6 (Liu [2]). Let $T>0$. Suppose A generates a pseudocontraction $C_{0}$-semigroup $S(t)$. That is, $\|S(t)\| \leq e^{\alpha t}$, $t \geq 0$, for some $\alpha \in \mathbb{R}$. Then the process $W_{A}^{\phi}(t)=\int_{0}^{t} S(t-$ $s) \phi(s) d W(s)$ has a continuous modification and there exists a constant $K>0$ such that

$$
E \sup _{s \in[0, t]}\left|W_{A}^{\phi}(s)\right|_{\mathbb{\square}}^{2} \leq K E \int_{0}^{t}|\phi(s)|_{\mathscr{L}_{2}^{0}}^{2} d s, \quad t \in[0, T] .
$$


Theorem 7. Let $\left(A_{1}\right)-\left(A_{4}\right)$ hold. Then the system (6) admits a unique mild solution $x(t) \in \mathscr{M}^{2}((-\infty, T], \mathbb{1})$ provided that

$$
0<\frac{8 M_{1-\alpha}^{2} M_{G}^{2} \gamma^{1-2 \alpha} \Gamma(2 \alpha-1)}{1-M_{G}\left\|(-A)^{-\alpha}\right\|}+2 M_{G}\left\|(-A)^{-\alpha}\right\|<1 .
$$

Proof. The proof is similar to the proof of Theorem 3.1 in Jiang and Shen [9], we omit the detail.

We now state our main theorem in this section.

Theorem 8. Let $\left(A_{1}\right)-\left(A_{3}\right),\left(A_{4}^{\prime}\right)$ and (21) hold. Then the system (6) admits a unique mild solution $x(t) \in \mathscr{M}^{2}((-\infty, T], \mathbb{U})$ provided that

$$
L:=4 M_{1-\alpha}^{2} M_{G}^{2} \gamma^{-2 \alpha} \Gamma(2 \alpha-1)+4 M_{G}^{2}\left\|(-A)^{-\alpha}\right\|^{2}<1 .
$$

Proof. Let $N$ be a positive integer and $T_{0} \in(0, T)$. We introduce the sequence of the functions $\left\{b^{N}(t, u)\right\}$ and $\left\{\sigma^{N}(t, u)\right\}$, $(t, u) \in[0, T] \times \mathscr{B}$ as follows:

$$
\begin{gathered}
b^{N}(t, u)= \begin{cases}b(t, u), & \|u\|_{\mathscr{B}} \leq N ; \\
b\left(t, \frac{N u}{\|u\|_{\mathscr{B}}}\right), & \|u\|_{\mathscr{B}}>N .\end{cases} \\
\sigma^{N}(t, u)= \begin{cases}\sigma(t, u), & \|u\|_{\mathscr{B}} \leq N ; \\
\sigma\left(t, \frac{N u}{\|u\|_{\mathscr{B}}}\right), & \|u\|_{\mathscr{B}}>N .\end{cases}
\end{gathered}
$$

Then the functions $\left\{b^{N}(t, u)\right\}$ and $\left\{\sigma^{N}(t, u)\right\}$ satisfy assumption $\left(A_{3}\right)$, and for any $x, y \in \mathscr{B}, t \in[0, T]$, the following inequality holds:

$$
\begin{aligned}
& \left|b^{N}(t, x)-b^{N}(t, y)\right|_{\mathscr{\varpi}}^{2}+\left|\sigma^{N}(t, x)-\sigma^{N}(t, y)\right|_{\mathscr{L}_{2}^{0}}^{2} \\
& \quad \leq Z^{N}\left(t,\|x-y\|_{\mathscr{B}}^{2}\right) .
\end{aligned}
$$

As a consequence of Theorem 7, there exist the unique mild solutions $x^{N}(t)$ and $x^{N+1}(t)$, respectively, to the following integral equations:

$$
\begin{aligned}
x^{N}(t)= & S(t)(\phi(0)+G(0, \phi))-G\left(t, x_{t}^{N}\right) \\
& -\int_{0}^{t} A S(t-s) G\left(s, x_{s}^{N}\right) d s \\
& +\int_{0}^{t} S(t-s) b^{N}\left(s, x_{s}^{N}\right) d s \\
& +\int_{0}^{t} S(t-s) \sigma^{N}\left(s, x_{s}^{N}\right) d W(s), \\
x^{N+1}(t)= & S(t)(\phi(0)+G(0, \phi))-G\left(t, x_{t}^{N+1}\right) \\
& -\int_{0}^{t} A S(t-s) G\left(s, x_{s}^{N+1}\right) d s \\
& +\int_{0}^{t} S(t-s) b^{N+1}\left(s, x_{s}^{N+1}\right) d s \\
& +\int_{0}^{t} S(t-s) \sigma^{N+1}\left(s, x_{s}^{N+1}\right) d W(s) .
\end{aligned}
$$

Define the stopping time

$$
\begin{aligned}
\delta_{N} & :=T_{0} \wedge \inf \left\{t \in[0, T]:\left\|x^{N}(t)\right\|_{\mathscr{B}} \geq N\right\}, \\
\delta_{N+1} & :=T_{0} \wedge \inf \left\{t \in[0, T]:\left\|x^{N+1}(t)\right\|_{\mathscr{B}} \geq N+1\right\}, \\
\tau_{N} & :=\delta_{N} \wedge \delta_{N+1} .
\end{aligned}
$$

In view of Hölder's inequality (25), we obtain

$$
\begin{aligned}
& E\left(\sup _{0 \leq s \leq \tau_{N} \wedge t}\left|x^{N+1}(s)-x^{N}(s)\right|_{\mathbb{H}}^{2}\right) \\
& \leq 4 E \sup _{0 \leq s \leq \tau_{N} \wedge t}\left|S(s)\left(G\left(s, x_{s}^{N+1}\right)-G\left(s, x_{s}^{N}\right)\right)\right|_{\mathbb{H}}^{2} \\
& \quad+4 E \sup _{0 \leq s \leq \tau_{N} \wedge t}\left|\int_{0}^{s} A S(s-u)\left(G\left(u, x_{u}^{N+1}\right)-G\left(u, x_{u}^{N}\right)\right) d u\right|_{\mathbb{H}}^{2} \\
& +4 E \sup _{0 \leq s \leq \tau_{N} \wedge t} \mid \int_{0}^{s} S(s-u) \\
& \quad+4 E \sup _{0 \leq s \leq \tau_{N} \wedge t} \mid \int_{0}^{s} S(s-u) \\
& \quad \times\left.\left(\sigma^{N+1}\left(u, x_{u}^{N+1}\right)-\sigma^{N+1}\left(u, x_{u}^{N}\right)\right) d W(u)\right|_{\mathbb{H}} ^{2} \\
& =4 \sum_{i=1}^{4} I_{i}, \quad
\end{aligned}
$$

where we have used the fact that for $0 \leq u \leq \tau_{N}$,

$$
\begin{aligned}
& b^{N+1}\left(u, x_{u}^{N}\right)=b^{N}\left(u, x_{u}^{N}\right), \\
& \sigma^{N+1}\left(u, x_{u}^{N}\right)=\sigma^{N}\left(u, x_{u}^{N}\right) .
\end{aligned}
$$

Note that

$$
\left\|x_{s}^{N+1}-x_{s}^{N}\right\|_{\mathscr{B}}^{2} \leq \sup _{0 \leq u \leq s}\left|x^{N+1}(u)-x^{N}(u)\right|_{\mathbb{H}}^{2} .
$$

By assumption $\left(A_{2}\right)$, we have

$$
\begin{aligned}
I_{1} & \leq\left\|(-A)^{-\alpha}\right\|^{2} M_{G}^{2} E \sup _{0 \leq s \leq \tau_{N} \wedge t}\left\|x_{s}^{N+1}-x_{s}^{N}\right\|_{\mathscr{B}}^{2} \\
& \leq\left\|(-A)^{-\alpha}\right\|^{2} M_{G}^{2} E \sup _{0 \leq s \leq \tau_{N} \wedge t}\left|x^{N+1}(s)-x^{N}(s)\right|_{\mathscr{H}}^{2} .
\end{aligned}
$$


By virtue of Lemma 5, Hölder's inequality together with assumption $\left(A_{2}\right)$ we have

$I_{2}$

$$
\begin{aligned}
& \leq \underset{0 \leq s \leq \tau_{N} \wedge t}{E} \sup _{0}\left[\int_{0}^{s}\left|A S(s-u)\left(G\left(u, x_{u}^{N+1}\right)-G\left(u, x_{u}^{N}\right)\right)\right|_{\mathbb{G}} d u\right]^{2} \\
& \leq M_{G}^{2} M_{1-\alpha}^{2} E \sup _{0 \leq s \leq \tau_{N} \wedge t} \int_{0}^{s}(s-u)^{2(\alpha-1)} e^{-\gamma(s-u)} d u \\
& \quad \times \int_{0}^{s} e^{-\gamma(s-u)}\left\|x_{u}^{N+1}-x_{u}^{N}\right\|_{\mathscr{B}}^{2} d u \\
& \leq M_{G}^{2} M_{1-\alpha}^{2} \gamma^{-2 \alpha} \Gamma(2 \alpha-1) E \sup _{0 \leq s \leq \tau_{N} \wedge t}\left|x^{N+1}(s)-x^{N}(s)\right|_{\mathbb{⿴}}^{2} .
\end{aligned}
$$

Employing assumption $\left(A_{4}^{\prime}\right)$, Hölder's inequality, and Jensen's inequality, it follows that

$$
\begin{aligned}
I_{3} \leq & E \sup _{0 \leq s \leq \tau_{N} \wedge t} \int_{0}^{s} e^{-2 \gamma(s-u)} d u \\
& \times \int_{0}^{s}\left|b^{N+1}\left(u, x_{u}^{N+1}\right)-b^{N+1}\left(u, x_{u}^{N}\right)\right|_{\mathbb{H}}^{2} d u \\
\leq & \frac{1}{2 \gamma} \int_{0}^{\tau_{N} \wedge t} E\left|b^{N+1}\left(s, x_{s}^{N+1}\right)-b^{N+1}\left(s, x_{s}^{N}\right)\right|_{\mathbb{H}}^{2} d s \\
\leq & \frac{1}{2 \gamma} \int_{0}^{t} Z_{N+1}\left(s \wedge \tau_{N}, E\left\|x_{s \wedge \tau_{N}}^{N+1}-x_{s \wedge \tau_{N}}^{N}\right\|_{\mathscr{B}}^{2}\right) d s .
\end{aligned}
$$

Combining Lemma 6 with Jensen's inequality, there exists a positive constant $K$ such that

$$
\begin{aligned}
I_{4} & \leq K \int_{0}^{\tau_{N} \wedge t} E\left|\sigma^{N+1}\left(s, x_{s}^{N+1}\right)-\sigma^{N+1}\left(s, x_{s}^{N}\right)\right|_{\mathscr{L}_{2}^{0}}^{2} d s \\
& \leq K \int_{0}^{t} Z_{N+1}\left(s \wedge \tau_{N}, E\left\|x_{s \wedge \tau_{N}}^{N+1}-x_{s \wedge \tau_{N}}^{N}\right\|_{\mathscr{B}}^{2}\right) d s .
\end{aligned}
$$

Therefore, for all $t \in\left[0, T_{0}\right]$, we have

$$
\begin{aligned}
& E\left(\sup _{0 \leq s \leq t}\left|x^{N+1}\left(s \wedge \tau_{N}\right)-x^{N}\left(s \wedge \tau_{N}\right)\right|_{\mathbb{Q}}^{2}\right) \\
& \leq \frac{4(1 / 2 \gamma+K)}{1-L} \int_{0}^{t} Z_{N+1}\left(s \wedge \tau_{N}, E\left\|x_{s \wedge \tau_{N}}^{N+1}-x_{s \wedge \tau_{N}}^{N}\right\|_{\mathscr{B}}^{2}\right) d s \\
& \leq \frac{4(1 / 2 \gamma+K)}{1-L} \\
& \times \int_{0}^{t} Z_{N+1}\left(s \wedge \tau_{N},\right. \\
& \left.\quad E \sup _{0 \leq u \leq s}\left|x^{N+1}\left(u \wedge \tau_{N}\right)-x^{N}\left(u \wedge \tau_{N}\right)\right|_{\mathbb{q}}^{2}\right) d s .
\end{aligned}
$$

The assumption $\left(A_{4}^{\prime}\right)$ indicates that

$$
E\left(\sup _{0 \leq s \leq t}\left|x^{N+1}\left(s \wedge \tau_{N}\right)-x^{N}\left(s \wedge \tau_{N}\right)\right|_{\mathbb{Q}}^{2}\right)=0 .
$$

Thus, for a.e. $\omega$,

$$
x^{N+1}(t)=x^{N}(t) \quad \text { for } 0 \leq t \leq T_{0} \wedge \tau_{N} .
$$

Note that for each $\omega \in \Omega$, there exists an $N_{0}(\omega)>0$ such that $0<T_{0} \leq \tau_{N_{0}}$. Define $x(t)$ by

$$
x(t)=x^{N_{0}}(t) \quad \text { for } t \in\left[0, T_{0}\right] .
$$

Since $x\left(t \wedge \tau_{N}\right)=x^{N}\left(t \wedge \tau_{N}\right)$, it holds that

$$
\begin{aligned}
x\left(t \wedge \tau_{N}\right)= & S\left(t \wedge \tau_{N}\right)(\phi(0)-G(0, \phi))+G\left(t \wedge \tau_{N}, x_{t \wedge \tau_{N}}^{N}\right) \\
& +\int_{0}^{t \wedge \tau_{N}} A S\left(t \wedge \tau_{N}-s\right) G\left(s, x_{s}^{N}\right) d s \\
& +\int_{0}^{t \wedge \tau_{N}} S\left(t \wedge \tau_{N}-s\right) b^{N}\left(s, x_{s}^{N}\right) d s \\
& +\int_{0}^{t \wedge \tau_{N}} S\left(t \wedge \tau_{N}-s\right) \sigma^{N}\left(s, x_{s}^{N}\right) d W(s) \\
= & S\left(t \wedge \tau_{N}\right)(\phi(0)-G(0, \phi))+G\left(t \wedge \tau_{N}, x_{t \wedge \tau_{N}}\right) \\
& +\int_{0}^{t \wedge \tau_{N}} A S\left(t \wedge \tau_{N}-s\right) G\left(s, x_{s}\right) d s \\
& +\int_{0}^{t \wedge \tau_{N}} S\left(t \wedge \tau_{N}-s\right) b\left(s, x_{s}\right) d s \\
& +\int_{0}^{t \wedge \tau_{N}} S\left(t \wedge \tau_{N}-s\right) \sigma\left(s, x_{s}\right) d W(s) .
\end{aligned}
$$

Taking $N \rightarrow \infty$, we have

$$
\begin{aligned}
x(t)= & S(t)(\phi(0)+G(0, \phi))-G\left(t, x_{t}\right) \\
& -\int_{0}^{t} A S(t-s) G\left(s, x_{s}\right) d s+\int_{0}^{t} S(t-s) f\left(s, x_{s}\right) d s \\
& +\int_{0}^{t} S(t-s) \sigma\left(s, x_{s}\right) d W(s),
\end{aligned}
$$

which completes the proof.

Remark 9. We obtain the existence and uniqueness of mild solution to (6) under local Carathéodory conditions with the non-Lipschitz conditions in $[9,18]$ being regarded as special cases, which makes it more feasible that the conditions of solution can be satisfied.

\section{Exponential Stability}

In this section, we consider the exponential stability in mean square and almost sure exponential stability of the mild 
solutions of (6). For the sake of brevity, we denote by $x^{\phi}$ or similar notations the unique mild solution of (6) with the initial data $\phi$.

Definition 10. The mild solution $x^{\phi}$ of (6) is said to be exponentially asymptotically stable in mean square if there exist a pair of positive constants $\alpha$ and $\beta$ such that, for any mild solution $y^{\varphi}$ of (6),

$$
E\left|x^{\phi}(t)-y^{\varphi}(t)\right|_{\mathbb{H}}^{2} \leq \alpha e^{-\beta t} E\|\phi-\varphi\|_{\mathscr{B}}^{2}, \quad t \geq 0 .
$$

We need the following assumptions before we proceed further.

$\left(A_{5}\right)$ For any $x, y \in \mathscr{B}$, there exist some positive constants $Q_{b}$ and $Q_{\sigma}$ such that

$$
\begin{gathered}
|b(t, x)-b(t, y)|_{[\mathbb{U}}^{2} \leq Q_{b}\|x-y\|_{\mathscr{B}}^{2}, \\
|\sigma(t, x)-\sigma(t, y)|_{\mathscr{L}_{2}^{0}}^{2} \leq Q_{\sigma}\|x-y\|_{\mathscr{B}}^{2},
\end{gathered}
$$

for all $t \geq 0$.

$\left(A_{6}\right)$ There exist some constant $\alpha \in(1 / 2,1]$ and a continuous function $\mu$ such that for any $\xi, \phi \in \mathscr{B}$, $t \geq 0$,

$$
\begin{gathered}
G(t, \xi), G(t, \phi) \in \mathscr{D}\left((-A)^{\alpha}\right), \\
\left|(-A)^{\alpha} G(t, \xi)-(-A)^{\alpha} G(t, \phi)\right| \leq \mu(t)\|\xi-\phi\|_{\mathscr{B}},
\end{gathered}
$$

where $\mu: \mathbb{R}^{+} \rightarrow \mathbb{R}^{+}$satisfies $\mu(t) \leq Q_{G} e^{-\delta t}, Q_{G}>0$, $\delta>\gamma>0$.

The following lemma is needed to consider our results.

Lemma 11 (see [16]). Assume that the semigroup $\{S(t), t \geq 0\}$ is exponentially stable; that is, $\|S(t)\| \leq M e^{-\gamma t}, t \geq 0$, for some $M, \gamma>0$. Then, for any $\mathscr{F}_{t}$-adapted predictable process $\Phi$ with $\int_{0}^{t} E|\Phi(s)|_{\mathscr{L}_{2}^{0}}^{2} d s<\infty, t \geq 0$, the following inequality holds:

$$
E\left|\int_{0}^{t} S(t-s) \Phi(s) d W(s)\right|_{\boxplus}^{2} \leq M^{2} \int_{0}^{t} e^{-\gamma(t-s)} E|\Phi(s)|_{\mathscr{L}_{2}^{0}}^{2} d s .
$$

Now, we state our main result of this section on the stability in mean square.

Theorem 12. Let $x^{\phi}$ and $y^{\varphi}$ be two mild solutions of (6) with the initial data $\phi$ and $\varphi$, respectively. Assume that $\left(A_{1}\right)$, $\left(A_{5}\right)-\left(A_{6}\right)$, and $5 Q_{G}^{2}\left\|(-A)^{-\alpha}\right\|^{2}<1$ hold. Then

$$
E\left(\sup _{0 \leq s \leq t} e^{\gamma s}|x(s)-y(s)|_{\mathbb{H}}^{2}\right) \leq \beta e^{\bar{\sigma} t} E\|\phi-\varphi\|_{\mathscr{B}}^{2}, \quad t \geq 0,
$$

where $\beta=10\left(1+Q_{G}^{2}\left\|(-A)^{-\alpha}\right\|^{2}\right) /\left(1-5 Q_{G}^{2}\left\|(-A)^{-\alpha}\right\|^{2}\right)$, $\bar{\sigma}=\left(5 M_{1-\alpha}^{2} Q_{G}^{2} \gamma^{1-2 \alpha} \Gamma(2 \alpha-1)+5 \gamma^{-1} Q_{b}+20 Q_{\sigma}\right) /(1-$ $\left.5 Q_{G}^{2}\left\|(-A)^{-\alpha}\right\|^{2}\right)$.
Proof. Since by assumption, $x=x^{\phi}$ and $y=y^{\varphi}$ are solutions of (6), we have

$$
\begin{aligned}
x(t)= & S(t)(\phi(0)+G(0, \phi))-G\left(t, x_{t}\right) \\
& -\int_{0}^{t} A S(t-s) G\left(s, x_{s}\right) d s+\int_{0}^{t} S(t-s) b\left(s, x_{s}\right) d s \\
& +\int_{0}^{t} S(t-s) \sigma\left(s, x_{s}\right) d W(s), \\
y(t)= & S(t)(\varphi(0)+G(0, \varphi))-G\left(t, y_{t}\right) \\
& -\int_{0}^{t} A S(t-s) G\left(s, y_{s}\right) d s+\int_{0}^{t} S(t-s) b\left(s, y_{s}\right) d s \\
& +\int_{0}^{t} S(t-s) \sigma\left(s, y_{s}\right) d W(s) .
\end{aligned}
$$

Then,

$$
\begin{aligned}
& E\left(\sup _{0 \leq s \leq t} e^{\gamma s}|x(s)-y(s)|_{\mathbb{H}}^{2}\right) \\
& \leq 5 E\left(\sup _{0 \leq s \leq t} e^{\gamma s}|S(s)[\phi(0)-\varphi(0)+G(0, \phi)-G(0, \varphi)]|_{\mathbb{H}}^{2}\right) \\
& +5 E\left(\sup _{0 \leq s \leq t} e^{\gamma s}\left|G\left(s, x_{s}\right)-G\left(s, y_{s}\right)\right|_{\mathbb{H}}^{2}\right) \\
& +5 E\left(\sup _{0 \leq s \leq t} e^{\gamma s}\left|\int_{0}^{s} A S(s-u)\left(G\left(u, x_{u}\right)-G\left(u, y_{u}\right)\right) d u\right|_{\mathbb{H}}^{2}\right) \\
& +5 E\left(\sup _{0 \leq s \leq t} e^{\gamma s}\left|\int_{0}^{s} S(s-u)\left(b\left(u, x_{u}\right)-b\left(u, y_{u}\right)\right) d u\right|_{\mathbb{H}}^{2}\right) \\
& +5 E\left(\sup _{0 \leq s \leq t} e^{\gamma s}\right. \\
& \left.\quad \times\left|\int_{0}^{s} S(s-u)\left(\sigma\left(u, x_{u}\right)-\sigma\left(u, y_{u}\right)\right) d W(u)\right|_{\mathbb{H}}^{2}\right) .
\end{aligned}
$$

We now estimate the terms on the right-hand side of (46). From assumption $\left(A_{1}\right)$ and $\left(A_{6}\right)$, we obtain

$$
\begin{aligned}
& \quad 5 E \sup _{0 \leq s \leq t} e^{\gamma s}|S(s)[\phi(0)-\varphi(0)+G(0, \phi)-G(0, \varphi)]|_{\mathbb{H}}^{2} \\
& \quad \leq 10 E \sup _{0 \leq s \leq t} e^{\gamma s} e^{-2 \gamma s}\left(|\phi(0)-\varphi(0)|_{\mathbb{H}}^{2}+|G(0, \phi)-G(0, \varphi)|_{\mathbb{H}}^{2}\right) \\
& \leq 10\left(1+Q_{G}^{2}\left\|(-A)^{-\alpha}\right\|^{2}\right) E\|\phi-\varphi\|_{\mathscr{B}}^{2} .
\end{aligned}
$$

Noting that $\delta>\gamma>0$ and

$$
\left\|x_{s}^{N+1}-x_{s}^{N}\right\|_{\mathscr{B}}^{2} \leq \sup _{0 \leq u \leq s}\left|x^{N+1}(u)-x^{N}(u)\right|_{\mathbb{U}^{\prime}}^{2}
$$


we have

$$
\begin{aligned}
& 5 E\left(\sup _{0 \leq s \leq t} e^{\gamma s}\left|G\left(s, x_{s}\right)-G\left(s, y_{s}\right)\right|_{\mathbb{W}}^{2}\right) \\
& \leq 5 Q_{G}^{2}\left\|(-A)^{-\alpha}\right\|^{2} E\left(\sup _{0 \leq s \leq t} e^{\gamma s} \mu(s)\left\|x_{s}-y_{s}\right\|_{\mathscr{B}}^{2}\right) \\
& \quad \leq 5 Q_{G}^{2}\left\|(-A)^{-\alpha}\right\|^{2} E\left(\sup _{0 \leq s \leq t} e^{\gamma s} e^{-2 \delta s}\left\|x_{s}-y_{s}\right\|_{\mathscr{B}}^{2}\right) \\
& \quad \leq 5 Q_{G}^{2}\left\|(-A)^{-\alpha}\right\|^{2} E\left(\sup _{0 \leq s \leq t} e^{\gamma s}|x(s)-y(s)|_{\mathbb{H}}^{2}\right) .
\end{aligned}
$$

Standard computations involving Hölder's inequality and Lemma 5 yield that

$$
\begin{gathered}
5 E\left(\sup _{0 \leq s \leq t} e^{\gamma s}\left|\int_{0}^{s} A S(s-u)\left(G\left(u, x_{u}\right)-G\left(u, y_{u}\right)\right) d u\right|_{\mathbb{H}}^{2}\right) \\
\leq 5 \sup _{0 \leq s \leq t} e^{\gamma s}\left[\int_{0}^{s} M_{1-\alpha}(s-u)^{\alpha-1} e^{-\gamma(s-u)} \mu(u)\right. \\
\left.\times\left\|x_{u}-y_{u}\right\|_{\mathscr{B}} d u\right]^{2} \\
\leq 5 M_{1-\alpha}^{2} Q_{G}^{2} E\left[\sup _{0 \leq s \leq t} e^{\gamma s} \int_{0}^{s}(s-u)^{2(\alpha-1)} e^{-\gamma(s-u)} d u\right. \\
\left.\quad \times \int_{0}^{s} e^{-\gamma(s-u)} e^{-2 \delta u}\left\|x_{u}-y_{u}\right\|_{\mathscr{B}}^{2} d u\right] \\
\leq 5 M_{1-\alpha}^{2} Q_{G}^{2} \gamma^{1-2 \alpha} \Gamma(2 \alpha-1) \\
\quad \times \int_{0}^{t} e^{\gamma s} E\left(\sup _{0 \leq r \leq s}|x(r)-y(r)|_{\mathbb{H}}^{2}\right) d s .
\end{gathered}
$$

Combing assumption $\left(A_{5}\right)$ with Hölder's inequality it follows that

$$
\begin{aligned}
5 E\left(\sup _{0 \leq s \leq t} e^{\gamma s}\left|\int_{0}^{s} S(s-u)\left(b\left(u, x_{u}\right)-b\left(u, y_{u}\right)\right) d u\right|_{\mathbb{H}}^{2}\right) \\
\leq 5 E \sup _{0 \leq s \leq t} e^{\gamma s} \int_{0}^{t} e^{-\gamma(s-u)} d u \\
\quad \times \int_{0}^{s} e^{-\gamma(s-u)}\left|b\left(u, x_{u}\right)-b\left(u, y_{u}\right)\right|_{\mathbb{H}}^{2} d u \\
\leq 5 \gamma^{-1} Q_{b} \int_{0}^{t} e^{\gamma s} E\left\|x_{s}-y_{s}\right\|_{\mathscr{B}}^{2} d s \\
\leq 5 \gamma^{-1} Q_{b} \int_{0}^{t} e^{\gamma s} E\left(\sup _{0 \leq r \leq s}\|x(r)-y(r)\|_{\mathbb{H}}^{2}\right) d s .
\end{aligned}
$$

Applying first Lemma 7.2 in [4] and then Lemma 11 we obtain

$$
\begin{aligned}
& \underset{0 \leq s \leq t}{5 E \sup ^{\gamma s}}\left|\int_{0}^{s} S(s-u)\left(\sigma\left(u, x_{u}\right)-\sigma\left(u, y_{u}\right)\right) d W(u)\right|_{\mathbb{E}}^{2} \\
& \quad \leq 20 \sup _{0 \leq s \leq t} e^{\gamma s} E\left|\int_{0}^{s} S(s-u)\left(\sigma\left(u, x_{u}\right)-\sigma\left(u, y_{u}\right)\right) d W(u)\right|_{\mathbb{W}}^{2} \\
& \quad \leq 20 Q_{\sigma} \int_{0}^{t} e^{\gamma s} E\left\|x_{s}-y_{s}\right\|_{\mathscr{B}}^{2} d s \\
& \quad \leq 20 Q_{\sigma} \int_{0}^{t} e^{\gamma s} E\left(\sup _{0 \leq r \leq s}\|x(r)-y(r)\|_{\mathbb{G}}^{2}\right) d s .
\end{aligned}
$$

Define

$$
\Lambda(t):=\underset{0 \leq s \leq t}{E \sup } e^{\gamma s}|x(s)-y(s)|_{\mathbb{H}}^{2} \quad \text { for } t \geq 0 .
$$

Recalling (46), from (47) to (52) we derive that

$$
\Lambda(t) \leq \beta E\|\phi-\varphi\|_{\mathscr{B}}^{2}+\bar{\sigma} \int_{0}^{t} \Lambda(s) d s,
$$

where $\beta=10\left(1+Q_{G}^{2}\left\|(-A)^{-\alpha}\right\|^{2}\right) /\left(1-5 Q_{G}^{2}\left\|(-A)^{-\alpha}\right\|^{2}\right)$, $\bar{\sigma}=\left(5 M_{1-\alpha}^{2} Q_{G}^{2} \gamma^{1-2 \alpha} \Gamma(2 \alpha-1)+5 \gamma^{-1} Q_{b}+20 Q_{\sigma}\right) /\left(1-5 Q_{G}^{2}\right.$ $\left.\left\|(-A)^{-\alpha}\right\|^{2}\right)$.

Invoking Gronwall's Lemma we get

$$
E\left(\sup _{0 \leq s \leq t} e^{\gamma s}|x(s)-y(s)|_{\mathscr{H}}^{2}\right) \leq \beta e^{\overline{\sigma t}} E\|\phi-\varphi\|_{\mathscr{B}}^{2}, \quad t \geq 0 .
$$

This completes the proof.

Corollary 13. Suppose that all the conditions of Theorem 12 hold. Then for any mild solutions $x^{\phi}$ and $y^{\varphi}$ of (6)

$$
E\left(\left|x^{\phi}(t)-y^{\varphi}(t)\right|_{\mathscr{H}}^{2}\right) \leq \beta e^{-(\gamma-\bar{\sigma}) t} E\|\phi-\varphi\|_{\mathscr{B}}^{2}, \quad t \geq 0,
$$

where $\beta$ and $\bar{\sigma}$ are defined in Theorem 12, $\gamma$ is the constant in assumption $\left(A_{1}\right)$. Consequently, if $\gamma>\bar{\sigma}$, then the mild solution $x^{\phi}$ is exponentially asymptotically stable in mean square.

Corollary 14. Suppose that all the conditions of Theorem 12 hold. If $G(t, 0)=b(t, 0)=\sigma(t, 0)=0$, for all $t \geq 0$ and $\gamma>$ $\bar{\sigma}$, then the trial solution of (6) is exponentially asymptotically stable in mean square.

Finally, we consider the stability of sample path.

Theorem 15. Suppose that all the conditions of Corollary 14 hold, then the sample path of the trial solution of (6) is exponentially asymptotically stable.

Proof. The method is similar to the proof of Theorem 5.1 in [7], we omit it here. 


\section{Conflict of Interests}

The authors declare that there is no conflict of interests regarding the publication of this paper.

\section{Acknowledgments}

The authors would like to express their sincere gratitude to the editor and the anonymous referees for their valuable comments and error corrections. Jing Cui is partially supported by the National Natural Science Foundation of China (11326171, 11271020), the Natural Science Foundation of Anhui Province (1208085MA11, 1308085QA14), the Key Natural Science Foundation of Anhui Educational Committee (KJ2013A133), and the PhD Start-up Fund of Anhui Normal University. Litan Yan is partially supported by National Natural Science Foundation of China (11171062) and Innovation Program of Shanghai Municipal Education Commission (12ZZ063). Xichao Sun is partially supported by the Natural Science Foundation of Anhui Province (1408085QA10).

\section{References}

[1] T. Caraballo, J. Real, and T. Taniguchi, "The exponential stability of neutral stochastic delay partial differential equations," Discrete and Continuous Dynamical Systems A, vol. 18, no. 2-3, pp. 295-313, 2007.

[2] K. Liu, Stability of Infinite Dimensional Stochastic Differential Equations with Applications, vol. 135 of Monographs and Surveys in Pure and Applied Mathematics, Chapman \& Hall/CRC, london, UK, 2006.

[3] J. Luo and K. Liu, "Stability of infinite dimensional stochastic evolution equations with memory and Markovian jumps," Stochastic Processes and Their Applications, vol. 118, no. 5, pp. 864-895, 2008.

[4] G. Da Prato and J. Zabczyk, Stochastic Equations in Infinite Dimensions, vol. 44 of Encyclopedia of Mathematics and Its Applications, Cambridge University Press, Cambridge, UK, 1992.

[5] S. Peszat and J. Zabczyk, Stochastic Partial Differential Equations with Lévy Noise, vol. 113 of Encyclopedia of Mathematics and Its Applications, Cambridge University Press, Cambridge, UK, 2007.

[6] F.-Y. Wang and T.-S. Zhang, "Gradient estimates for stochastic evolution equations with non-Lipschitz coefficients," Journal of Mathematical Analysis and Applications, vol. 365, no. 1, pp. 1-11, 2010.

[7] T. E. Govindan, "Almost sure exponential stability for stochastic neutral partial functional differential equations," Stochastics, vol. 77, no. 2, pp. 139-154, 2005.

[8] T. Taniguchi, K. Liu, and A. Truman, "Existence, uniqueness, and asymptotic behavior of mild solutions to stochastic functional differential equations in Hilbert spaces," Journal of Differential Equations, vol. 181, no. 1, pp. 72-91, 2002.

[9] F. Jiang and Y. Shen, "A note on the existence and uniqueness of mild solutions to neutral stochastic partial functional differential equations with non-Lipschitz coefficients," Computers \& Mathematics with Applications, vol. 61, no. 6, pp. 1590-1594, 2011.

[10] A. M. Samoilenko, N. I. Mahmudov, and A. N. Stanzhitskii, "Existence, uniqueness, and controllability results for neutral
FSDES in Hilbert spaces," Dynamic Systems and Applications, vol. 17, no. 1, pp. 53-70, 2008.

[11] M. E. Gurtin and A. C. Pipkin, "A general theory of heat conduction with finite wave speeds," Archive for Rational Mechanics and Analysis, vol. 31, no. 2, pp. 113-126, 1968.

[12] J. W. Nunziato, "On heat conduction in materials with memory," Quarterly of Applied Mathematics, vol. 29, pp. 187-204, 1971.

[13] Y. Ren and D. D. Sun, "Second-order neutral stochastic evolution equations with infinite delay under Carathéodory conditions," Journal of Optimization Theory and Applications, vol. 147, no. 3, pp. 569-582, 2010.

[14] Y. Li and B. Liu, "Existence of solution of nonlinear neutral stochastic differential inclusions with infinite delay," Stochastic Analysis and Applications, vol. 25, no. 2, pp. 397-415, 2007.

[15] J. Cui and L. Yan, "Asymptotic behavior for neutral stochastic partial differential equations with infinite delays," Electronic Communications in Probability, vol. 18, no. 45, pp. 1-12, 2013.

[16] T. Taniguchi, "Asymptotic stability theorems of semilinear stochastic evolution equations in Hilbert spaces," Stochastics and Stochastics Reports, vol. 53, no. 1-2, pp. 41-52, 1995.

[17] J. K. Hale and J. Kato, "Phase space for retarded equations with infinite delay," Funkcialaj Ekvacioj, vol. 21, no. 1, pp. 11-41, 1978.

[18] J. Bao and Z. Hou, "Existence of mild solutions to stochastic neutral partial functional differential equations with nonLipschitz coefficients," Computers \& Mathematics with Applications, vol. 59, no. 1, pp. 207-214, 2010.

[19] A. Pazy, Semigroups of Linear Operators and Applications to Partial Differential Equations, vol. 44 of Applied Mathematical Sciences, Springer, New York, NY, USA, 1983. 


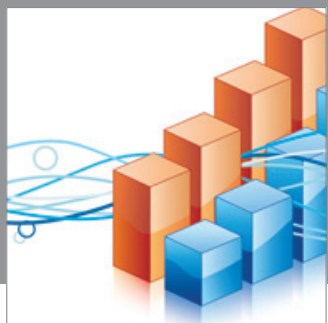

Advances in

Operations Research

mansans

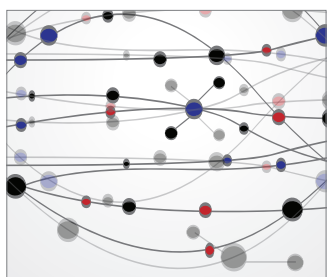

The Scientific World Journal
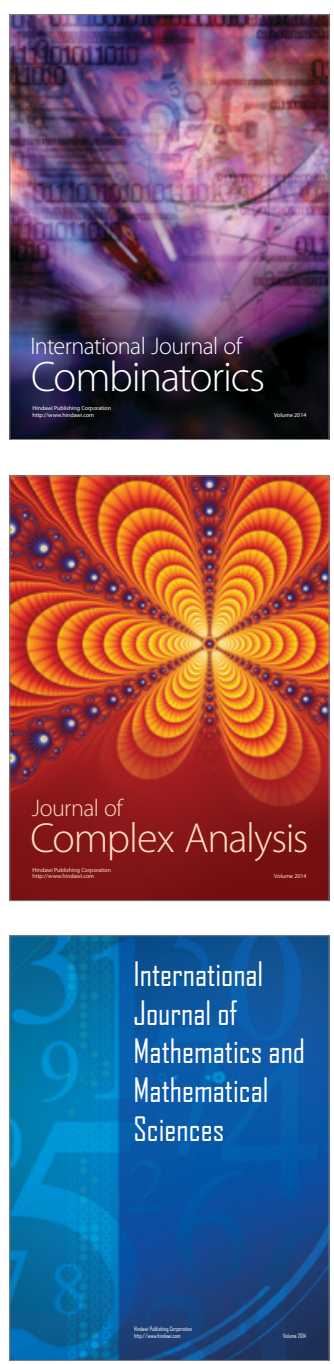
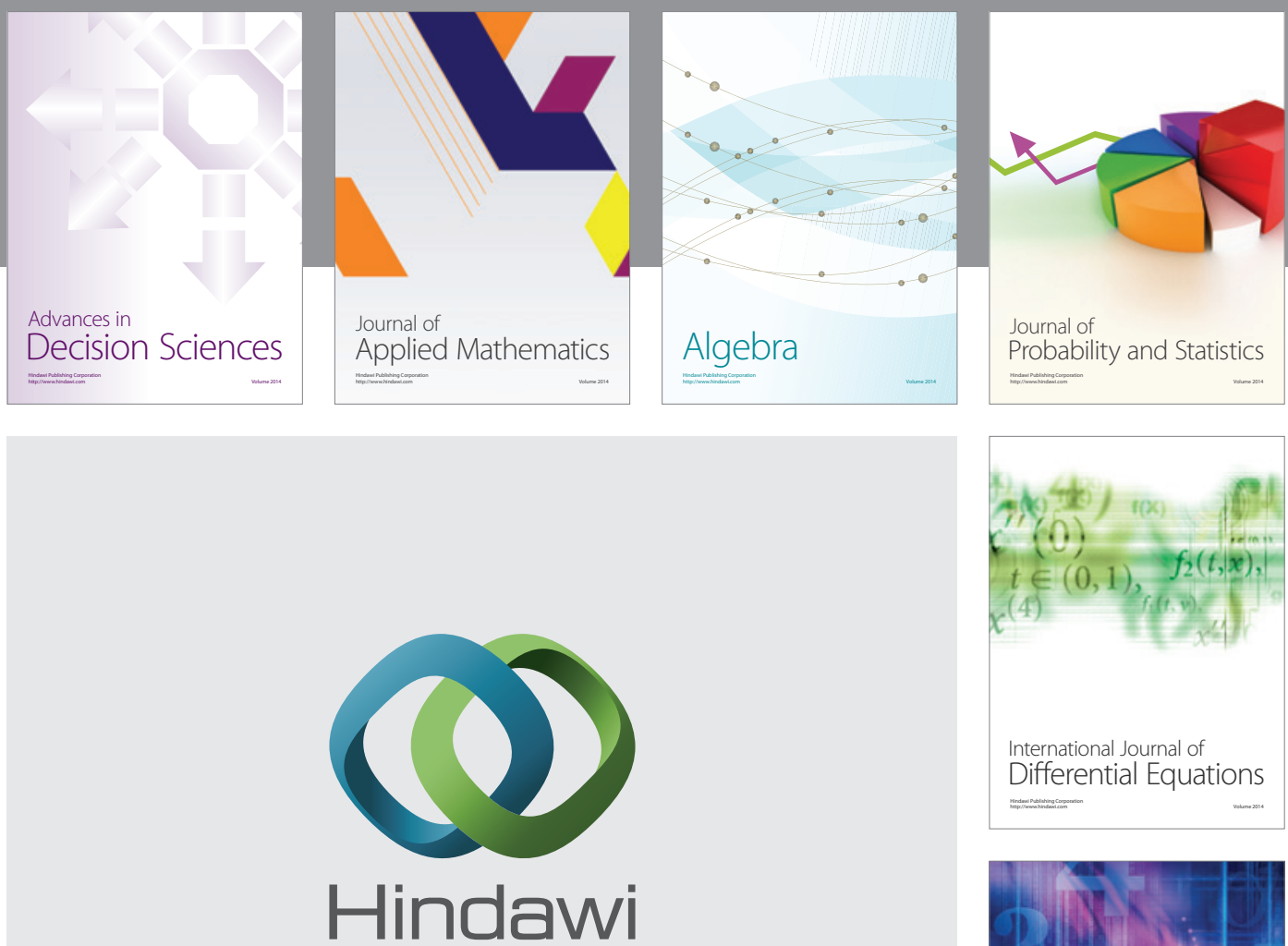

Submit your manuscripts at http://www.hindawi.com
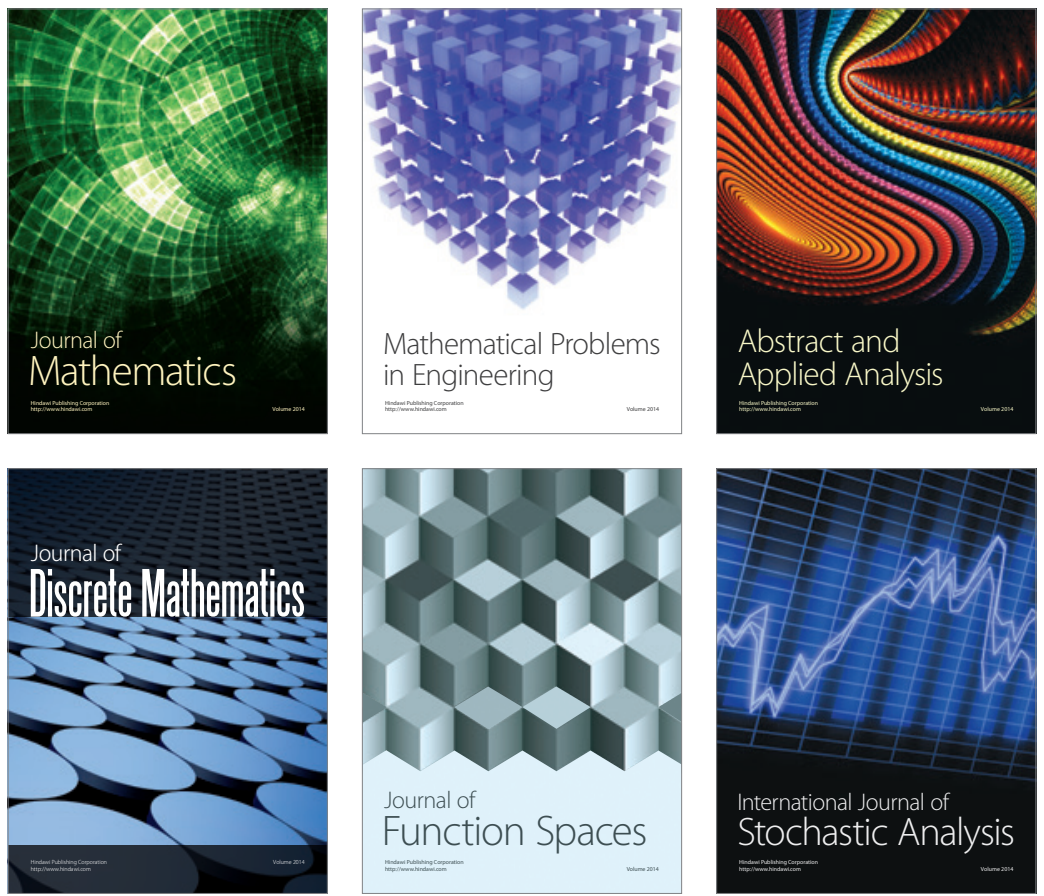

Journal of

Function Spaces

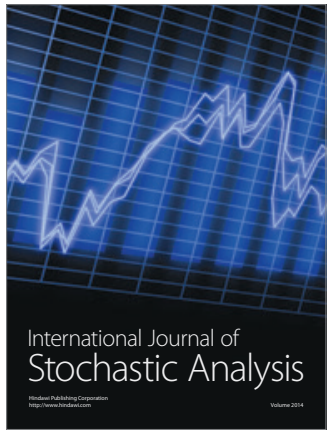

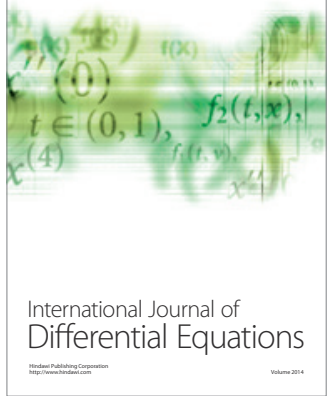
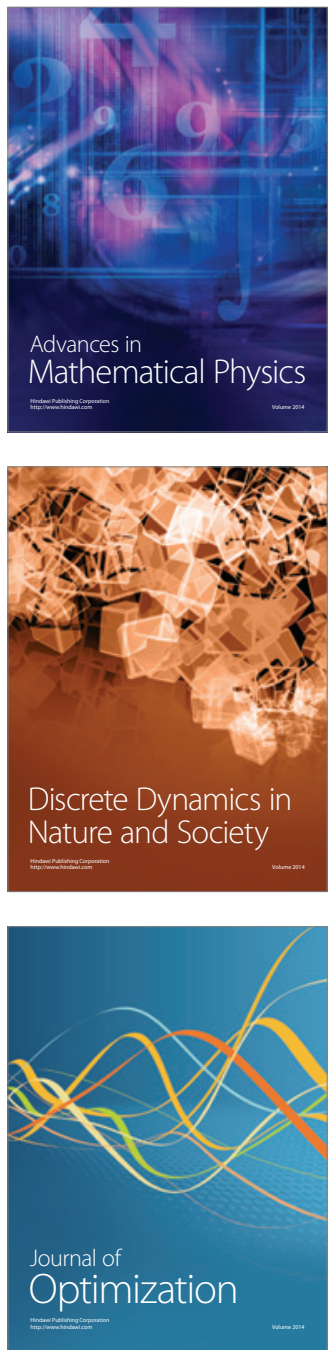